\title{
Human PRP4 kinase is required for stable tri-snRNP association during spliceosomal B complex formation
}

\author{
Marc Schneider $^{1}$, He-Hsuan Hsiao ${ }^{2}$, Cindy L Will ${ }^{1}$, Régis Giet $^{3}$, Henning Urlaub $^{2}$ \& Reinhard Lührmann ${ }^{1}$ \\ Reversible protein phosphorylation has an essential role during pre-mRNA splicing. Here we identify two previously \\ unidentified phosphoproteins in the human spliceosomal B complex, namely the pre-mRNA processing factors PRP6 and \\ PRP31, both components of the U4/U6-U5 tri-small nuclear ribonucleoprotein (snRNP). We provide evidence that PRP6 and \\ PRP31 are directly phosphorylated by human PRP4 kinase (PRP4K) concomitant with their incorporation into B complexes. \\ Immunodepletion and complementation studies with HeLa splicing extracts revealed that active human PRP4K is required for the \\ phosphorylation of PRP6 and PRP31 and for the assembly of stable, functional B complexes. Thus, the phosphorylation of PRP6 \\ and PRP31 is likely to have a key role during spliceosome assembly. Our data provide new insights into the molecular mechanism \\ by which PRP4K contributes to splicing. They further indicate that numerous phosphorylation events contribute to spliceosome \\ assembly and, thus, that splicing can potentially be modulated at multiple regulatory checkpoints.
}

Pre-mRNA splicing is catalyzed by the spliceosome, a dynamic molecular machine formed by the ordered interaction of the U1, U2, U4/U6 and U5 snRNPs, plus many non-snRNP proteins (reviewed in ref. 1). U1 and U2 interact first with the pre-mRNA to generate the spliceosomal A complex, which is then converted to the pre-catalytic B complex by the stable association of the preformed U4/U6-U5 tri-snRNP. After dramatic rearrangements, including destabilization of $\mathrm{U} 1$ and $\mathrm{U} 4$, the B complex is activated. The first catalytic step of splicing, which involves cleavage at the pre-mRNA's $5^{\prime}$ splice site and ligation of the intron's $5^{\prime}$ end to the branch site, subsequently ensues, generating the $\mathrm{C}$ complex. The $\mathrm{C}$ complex then catalyzes the second step of splicing, during which the $3^{\prime}$ splice site is cleaved and the $5^{\prime}$ and $3^{\prime}$ exon are ligated to form mRNA.

Protein-protein interactions have a key role in tri-snRNP formation and tri-snRNP integration into the spliceosome. In humans, at least two proteins mediate tri-snRNP formation, namely the U4/U6-associated PRP31 protein (also known as PRPF31) and the U5-associated PRP6 protein (also known as PRPF6) 2,3 . Human PRP31 is an essential splicing factor ${ }^{2}$, and specific mutations in PRP31 are associated with autosomal dominant retinitis pigmentosa ${ }^{4}$. Stable integration of the human tri-snRNP into the spliceosome requires several proteins, including PRP28 (also known as DDX23) ${ }^{5}$, the tri-snRNP proteins SAD1 (also known as USP39) and SNU66 (also known as SART1) ${ }^{6}$, the U2-associated SPF30 (also known as SMNDC1) protein ${ }^{7,8}$ and SR (serine-arginine-rich) proteins ${ }^{9}$.

Reversible protein phosphorylation affects most cellular activities in eukaryotes, including pre-mRNA splicing. Both the phosphorylation and dephosphorylation of spliceosomal proteins are important during the splicing process ${ }^{5,10-15}$. Known human spliceosomal and snRNP phosphoproteins include members of the SR protein family ${ }^{16,17}$, U1-70K (also known as SNRNP70) ${ }^{18}$, SF3B155 (also known as SF3B1) ${ }^{19}$, PRP28 (ref. 5) and several U4/U6-U5 tri-snRNP proteins ${ }^{20}$. SR proteins facilitate the association of snRNPs with the pre-mRNA during spliceosome assembly, and phosphorylation is essential for SR protein activity ${ }^{16,21}$ and enhances their interactions with other spliceosomal proteins ${ }^{13}$. Human PRP28, a U5 snRNP protein, is phosphorylated by the protein kinase SRPK2, and this event is a prerequisite for tri-snRNP addition during B complex formation ${ }^{5}$. The U2-associated SF3B155 protein is specifically hyperphosphorylated just before step I of splicing ${ }^{19}$ and is dephosphorylated by the PP1 or PP2A phosphatases during step II of splicing ${ }^{15}$.

Several protein kinases are involved in splicing, although in many cases, their specific targets are not well understood. Kinases known to phosphorylate the RS-rich regions of SR proteins and other spliceosomal proteins include members of the Clk/Sty family ${ }^{22}$, DNA topoisomerase 1 (ref. 23) and members of the SRPK family ${ }^{24-26 .}$ Prp4 kinase $(\operatorname{Prp} 4 \mathrm{~K})$ is essential for pre-mRNA splicing in Schizosaccharomyces pombe ${ }^{27}$, but the molecular mechanism of Prp4K action and the stage of splicing at which it acts are unknown. Prp4K belongs to the Clk/Sty family ${ }^{28}$ and is a Ser/Thr kinase. In higher eukaryotes, Prp4K contains, in addition to its highly conserved kinase domain, a neighboring RS domain and an N-terminal lysine-rich domain, both of which are lacking in $S$. pombe $e^{29}$. Prp $4 \mathrm{~K}$ can phosphorylate itself ${ }^{29}$, the SR protein ASF (also known as SF2) 30,31 and Prp6 (refs. 29,32). Mammalian and S. pombe Prp4K interact physically with Prp6 (refs. 29,32). Furthermore, S. pombe Prp4K interacts genetically with the proteins Prp 8 and Brr2, which facilitate spliceosome activation ${ }^{33}$. Thus, Prp4K was proposed to have a role in the formation of catalytically active spliceosomes ${ }^{33}$, although formal proof for this is currently lacking. Prp4K is also involved in

${ }^{1}$ Department of Cellular Biochemistry and ${ }^{2}$ Bioanalytical Mass Spectrometry Group, Max Planck Institute of Biophysical Chemistry, Göttingen, Germany. ${ }^{3}$ Institut de Génétique et Développement de l'Université de Rennes I, Rennes, France. Correspondence should be addressed to R.L. (reinhard.Iuehrmann@mpi-bpc.mpg.de). 
Figure 1 Human PRP6 and PRP31 are phosphorylated in spliceosomal B complexes. (a) Proteins isolated from purified human U4/ U6-U5 tri-snRNPs (lane 1), spliceosomal B complexes (lane 3 ) or a mixture of both (lane 2) were separated by SDS-PAGE and subsequently analyzed by western analysis with antibodies to human PRP6, PRP31 or SNU66, as indicated. (b) Schematic representation of human PRP6 and PRP31, showing known domains and the positions of phosphorylation sites, as identified by MS. (c) Antibodies recognizing a phosphoepitope of either human PRP6 or PRP31 were generated by immunizing rabbits with phosphorylated peptides (see Online Methods). Proteins isolated from U4/U6-U5 tri-snRNPs (lanes 1 and 3) or purified spliceosomal B complexes (lanes 2 and 4) were separated by SDS-PAGE and analyzed by western blotting with antibodies recognizing all forms of PRP6 or PRP31 (lanes 1 and 2) or antibodies recognizing solely phosphorylated PRP6 or PRP31 (lanes 3 and 4). Note that less B complex was analyzed in $\mathbf{c}$ than in $\mathbf{a}$; thus, some modified species were not readily detected. mitosis ${ }^{34}$ and was implicated in coupling pre-mRNA splicing with chromatin-remodeling events that regulate transcription ${ }^{29}$.

Here we demonstrate that the human tri-snRNP-associated proteins PRP6 and PRP31 are phosphorylated during spliceosomal $\mathrm{B}$ complex formation and, further, that their phosphorylation, as well as pre-mRNA splicing, is dependent on human PRP4K (also known as PRPF4B). We also demonstrate that stable association of the tri-snRNP during B complex formation is dependent on PRP4K, suggesting that PRP6 and PRP31 phosphorylation by this kinase has a key role in promoting tri-snRNP integration during spliceosome assembly.

\section{RESULTS}

\section{PRP6 and PRP31 are modified during spliceosome assembly}

To screen for proteins potentially modified during splicing, we initially performed immunoblotting with proteins isolated from human snRNPs or from purified spliceosomal B complexes. We detected a single band in purified tri-snRNPs by antibodies specific for human SNU66, PRP6 and PRP31 (Fig. 1a), but we observed multiple, slower-migrating bands with antibodies against PRP6 and PRP31 when proteins from purified B complexes were analyzed. This indicates that human PRP6 and PRP31 are modified during spliceosome assembly and that B complexes predominantly contain modified forms of these proteins.

\section{Identification of phosphorylated PRP6 and PRP31 residues}

MS analyses revealed that, in purified B complexes, human PRP6 and PRP31 are phosphorylated at multiple sites. In PRP6, we detected phosphoserines at positions 263 and 279, and phosphothreonines at positions 205, 266 and 275 (Fig. 1b and Supplementary Fig. 1). Most of these sites are clustered just upstream of the TPR repeat region of PRP6. An alignment of the phosphorylated region of human PRP6 with homologs from Mus musculus, Drosophila melanogaster, Caenhorhabditis elegans, S. pombe and Saccharomyces cerevisiae
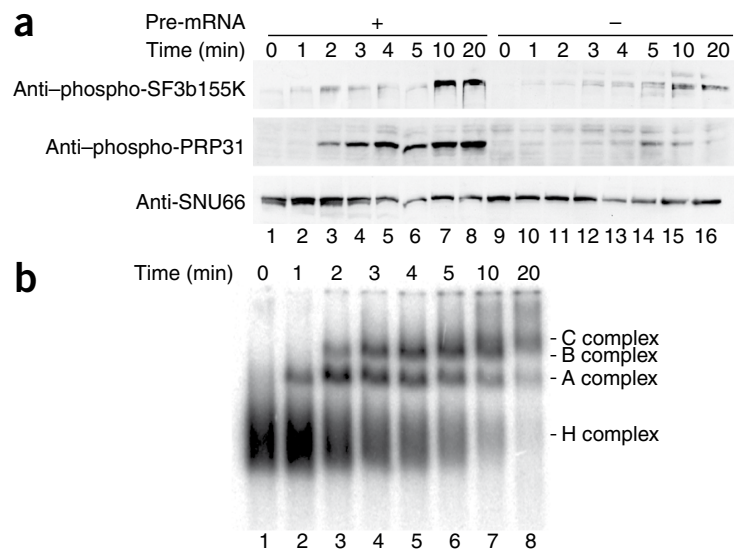

revealed that, with the exception of Prp6 in S. cerevisiae, Thr275 and Ser279 (amino acid positions according to human PRP6) are 100\% conserved (Supplementary Fig. 2). Ser263 and Thr266 are also conserved, although in some organisms serine is replaced by threonine and vice versa. In human PRP31, we detected phosphoserines at positions 439,498 and either 445 or 446 (MS could not distinguish between these two sites) and potentially at serines 450 or 451 (a conclusive assignment at either of these sites could not be made), and we found phosphothreonines at positions 440 and 455 (Fig. 1b and Supplementary Fig. 1). Similar to PRP6, most of these residues (except Ser445, Ser446 and Ser498) are highly conserved across evolution, with the notable exception of S. cerevisiae (Supplementary Fig. 2). This suggests that these amino acids have an important function and that they are phosphorylated not only in humans but also in a wide variety of organisms.

To analyze human PRP6 and PRP31 phosphorylation in more detail, we raised antibodies against phosphorylated peptides comprising amino acids $271-279$ of PRP6 or 434-453 of PRP31, containing the above phosphorylation sites. Each of these antibodies recognized a band that migrated slower than the one detected in purified tri-snRNPs by antibodies directed against a nonphosphorylated region of PRP6 or PRP31 (Fig. 1c). Thus, at least one of the slower-migrating PRP6 and PRP31 species observed in purified B complexes is phosphorylated.

\section{PRP31 phosphorylation requires spliceosome formation}

We next performed splicing in vitro with HeLa nuclear extract and followed the kinetics of PRP31 phosphorylation via western blotting. Owing to the lower activity of the anti-phospho-PRP6 antibodies, it was not possible to unequivocally detect phosphorylated PRP6 in nuclear extract. In the presence of adenovirus-derived MINX premRNA, PRP31 was first phosphorylated after 2 min (Fig. 2a, lane 3), which correlates with the appearance of the B complex (Fig. 2b, lane 3). The amount of phosphorylated PRP31 increased before reaching a plateau after $4 \mathrm{~min}$. When no pre-mRNA substrate was added, we observed only a low level of phospho-PRP31 (Fig. 2a, lanes 9-16). We first detected phosphorylation of SF3B155, which occurs after B complex formation $^{19}$, after 10 min of splicing, coinciding with the appearance of the C complex (Fig. 2a,b, lane 7). Thus, phosphorylation

Figure 2 Phosphorylation of human PRP31 is dependent on formation of the spliceosomal B complex and precedes SF3B155 phosphorylation. (a) In vitro splicing was performed with HeLa nuclear extract with (lanes 1-8) or without (lanes 9-16) exogenously added MINX pre-mRNA substrate for the indicated times. Proteins were subsequently analyzed by western blotting with antibodies specific for phosphorylated SF3B155 or phosphorylated PRP31, or as a loading control with an antibody against SNU66, as indicated at left. (b) Spliceosome assembly was analyzed at the indicated time points on an agarose gel and visualized by autoradiography. The position of the $\mathrm{H}, \mathrm{A}, \mathrm{B}$ and $\mathrm{C}$ complexes is indicated. 
a

Nuclear extract Mock $\triangle \mathrm{PRP} 4 \mathrm{~K}$

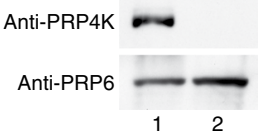

C
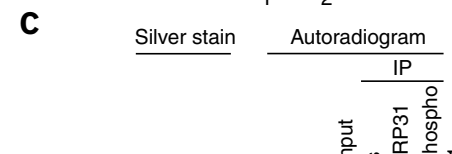

b Nuclear extract Mock $\triangle$ PRP4K Rec. PRP4K Anti-phospho-PRP6

Anti-PRP

Anti-phospho-PRP31 Anti-PRP31

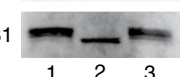

d

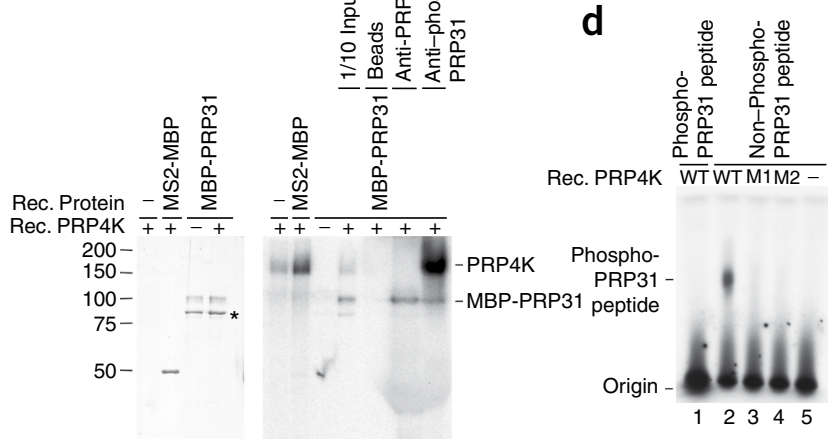

$25-$

$1234 \quad 5678 \quad 91011$ of PRP31 in HeLa nuclear extract is dependent on spliceosome assembly and temporally precedes SF3B 155 phosphorylation.

\section{Phosphorylation of PRP6 and PRP31 is dependent on PRP4K}

Our data suggest that tri-snRNP integration into the spliceosome is accompanied by phosphorylation of human PRP6 and PRP31. To dissect the role of PRP4K in PRP6 and PRP31 phosphorylation, and also in splicing, we first immunodepleted it from HeLa nuclear extract under high-salt conditions. Western blot analysis of mock-depleted versus $\mathrm{PRP} 4 \mathrm{~K}$-depleted $(\triangle \mathrm{PRP} 4 \mathrm{~K})$ extract revealed quantitative depletion of PRP4K (Fig. 3a).

Owing to residual antibody in the $\triangle \mathrm{PRP} 4 \mathrm{~K}$ extract, it was not possible via western analysis to directly assay the phosphorylation status of PRP31, as it co-migrates with the immunoglobulin G (IgG) heavy chain. Thus, to determine whether phosphorylation of PRP6 and/or PRP31 was affected, we first performed splicing in mock-depleted or $\triangle \mathrm{PRP} 4 \mathrm{~K}$ extract with MINX pre-mRNA containing aptamers of the MS2 phage coat protein (pre-bound with MS2-maltose-binding protein (MBP)). We then affinity selected spliceosomal complexes and analyzed them by western blotting. Whereas phosphorylated PRP6 and PRP31 were present in complexes formed in the mockdepleted extract, we no longer observed them in $\triangle \mathrm{PRP} 4 \mathrm{~K}$ extract (Fig. 3b, lanes 1 and 2). However, we detected nonphosphorylated PRP6 and PRP31 in both cases (Fig. 3b, lanes 1 and 2), suggesting that these proteins (presumably in the form of the tri-snRNP) are still recruited to spliceosomes. To confirm that PRP4K is responsible for the altered phosphorylation status of human PRP6 and PRP31, we overexpressed His-tagged wild-type PRP4K, as well as two mutated forms with single-residue changes in the ATP-binding site (M1 with a K717I mutation) or predicted active site (M2 with a D815A mutation) (Supplementary Fig. 3). Upon complementation of the $\triangle \mathrm{PRP} 4 \mathrm{~K}$ extract with purified wild-type PRP4K, but not an inactive form of PRP4K, we detected phosphorylated PRP6 and PRP31 in the affinityselected complexes (Fig. 3b, lane 3, and Supplementary Fig. 4). Thus, the phosphorylation of both proteins in splicing extract is dependent on PRP4K activity.
Figure 3 Phosphorylation of human PRP6 and PRP31 is dependent on PRP4 kinase. (a) Human PRP4 kinase was immunodepleted from Hela nuclear extract (see Online Methods). Proteins from mock-depleted (lane 1 ) or PRP4K-depleted ( $\triangle$ PRP4K) extract (lane 2) were separated by SDS-PAGE, blotted to nitocellulose and probed with anti-PRP4K or anti-PRP6 (loading control) antibodies, as indicated. (b) Western blot analysis of MS2 affinity-purified splicing complexes formed in mockdepleted nuclear extract (lane 1), $\triangle$ PRP4K nuclear extract (lane 2) or $\triangle$ PRP4K complemented with recombinant (Rec.) human PRP4K (lane 3). Western blots were probed with phosphospecific antibodies against PRP6 or PRP31, or with antibodies recognizing all forms of PRP6 and PRP31, as indicated at left. (c) PRP31 is phosphorylated in vitro by human PRP4K. MS2-MBP (lanes 2 and 6) or MBP-PRP31 (lanes 3, 4, and 7-11) were incubated with $\left(\gamma^{32}\right.$ P)ATP in the presence or absence of recombinant PRP4K (as indicated above each lane). The silver-stained gel (lanes 1-4) and corresponding autoradiograph (lanes 5-8) are shown. In lanes 9 and 10, immunoprecipitation (IP) of the phosphorylated protein in lane 8 (which shows one-tenth of the IP input) was performed with beads alone as a background binding control (lane 9), or with antibodies against both phosphorylated and nonphosphorylated PRP31 (lane 10) or phosphospecific anti-PRP31 antibodies (lane 11), and visualized solely by autoradiography. A co-purifying contaminant of unknown identity is indicated by an asterisk. (d) Thin-layer chromatography of the phosphorylated (lane 1) and nonphosphorylated (lanes 2-5) human PRP31 peptide (residues 434-453) incubated with the His-tagged wildtype (WT) human PRP4K or inactive mutants thereof (M1 and M2), as indicated. Phosphorylated peptides were visualized by autoradiography.

\section{Purified human PRP4K phosphorylates PRP31 in vitro}

Human PRP4K was previously shown to directly phosphorylate PRP6 in vitro and also to phosphorylate itself $f^{29,32}$. To test whether PRP31 is also a direct target of this kinase, we performed in vitro phosphorylation studies with purified PRP4K and recombinant PRP31 fused to MBP. Consistent with previous reports, incubation of purified PRP4K with $\left(\gamma_{-}{ }^{32} \mathrm{P}\right)$ ATP led to the phosphorylation of an $\sim 150-\mathrm{kDa}$ protein, which probably corresponds to autophosphorylated PRP4K (Fig. 3c, lane 5). In contrast, we did not observe additional ${ }^{32} \mathrm{P}$-labeled bands when we incubated purified PRP4K with MS2-MBP, demonstrating that MBP is not phosphorylated by this kinase (Fig. 3c, lane 6). Upon addition of purified PRP4K to MBPPRP31, we observed radiolabeled bands migrating at the position of MPB-PRP31 $(\sim 100 \mathrm{kDa})$ and at the position of autophosphorylated PRP4K (Fig. 3c, lane 8). The radiolabeled 100-kDa protein was precipitated by both anti-PRP31 and anti-phospho-PRP31 antibodies, but was not precipitated in the absence of antibody (Fig. 3c, lanes 9-11), confirming that PRP31 was phosphorylated. Phosphorylated PRP4K was also precipitated by the anti-phospho-PRP31 antibodies, suggesting that they cross-react with PRP4K or, alternatively, that phosphorylated PRP4K associates with phosphorylated PRP31 and is thereby co-precipitated.

In vitro phosphorylation with a nonphosphorylated or phosphorylated PRP31 peptide encompassing amino acids 434-453 (VYGGKS ${ }^{\mathrm{P}}$ $T^{\mathrm{P}}$ IRDRSSGT ${ }^{\mathrm{P} A S S V A}$, where $\mathrm{P}$ indicates phosphorylation), confirmed that wild-type PRP4K, but neither of the PRP4K mutants, specifically phosphorylates in vitro one or more of the phosphoserines and phosphothreonines detected by MS (Fig. 3d). Taken together, these studies demonstrate that PRP31 is a substrate of human PRP4K in vitro and, together with PRP6, is therefore probably directly phosphorylated by this kinase in HeLa nuclear extract under splicing conditions.

\section{B complex formation is reduced in $\triangle \mathrm{PRP} 4 \mathrm{~K}$ extract}

To determine the role of human PRP4K in splicing, we performed an in vitro splicing assay with MINX pre-mRNA and either mock-depleted or $\triangle \mathrm{PRP} 4 \mathrm{~K}$ nuclear extract. Both steps of splicing were clearly reduced 
Figure 4 Human PRP4 kinase is required for spliceosomal B complex formation. (a) In vitro splicing of ${ }^{32} \mathrm{P}$-labeled MINX pre-mRNA in mockdepleted nuclear extract (lanes 1 and 2), PRP4K-depleted ( $\triangle \mathrm{PRP} 4 \mathrm{~K}$ ) nuclear extract (lane 3 ) or $\triangle$ PRP4K complemented with recombinant His-tagged wild-type PRP4K (Rec.; lane 4) or mutant PRP4K (M1 or M2) (lanes 5 or 6 ). RNA was analyzed on a $7 \mathrm{M}$ urea-10\% polyacrylamide gel and visualized by autoradiography. The positions of the pre-mRNA and splicing intermediates and products are indicated at left. Note that the double bands observed with some RNA species are due to heterogeneity at the $3^{\prime}$ end of the in vitro-transcribed pre-mRNA. The ratio of mRNA to pre-mRNA was quantified with a Phosphorlmager and is indicated below each lane. (b) Spliceosome assembly in mock-depleted nuclear extract (lanes 1 and 2), $\triangle$ PRP4K nuclear extract (lane 3) or $\triangle$ PRP4K complemented with recombinant His-tagged wild-type PRP4K (lane 4) or mutant PRP4K (M1 or M2) (lanes 5 or 6 ) was analyzed on an agarose gel and visualized by autoradiography. The positions of the $H, A$ and $B$ complexes are indicated. The ratio of $B$ to $A$ complex was quantified with a Phosphorlmager and is indicated below each lane.

in $\triangle \mathrm{PRP} 4 \mathrm{~K}$ extract as compared to the mock-depleted extract (Fig. 4a, lanes 2 and 3). Notably, splicing could be restored by addition of purified wild-type PRP4K but not by inactivated PRP4K (that is, M1 or M2) (Fig. 4a, lanes 4-6), consistent with previous studies in S. pombe indicating a requirement for active Prp4K in splicing ${ }^{32,33}$. We obtained similar results with a $\beta$-globin pre-mRNA substrate (Supplementary Fig. 5), demonstrating that the inhibitory effect of PRP4K depletion is not specific for the MINX pre-mRNA. Thus, similar to its $S$. pombe counterpart, human PRP4K is required for splicing in vitro.

To determine at what step of splicing human PRP4K acts, we analyzed splicing complex formation. Relative to mock-depleted extract, we observed a clear decrease in B complex formation, with a corresponding increase in A complex, when splicing was performed with the $\triangle \mathrm{PRP} 4 \mathrm{~K}$ extract (Fig. 4b, lanes 2 and 3). Furthermore, we could restore B complex formation by adding wild-type, but not mutant, PRP4K (Fig. 4b, lanes 4-6). These data clearly demonstrate a role for human PRP4K in spliceosome assembly at the stage of tri-snRNP addition.

\section{Tri-snRNP levels are not affected by PRP4K depletion}

Both human PRP6 and PRP31 have essential roles in the stability of the tri-snRNP in vivo ${ }^{3}$ and in vitro ${ }^{2}$. As these proteins are no longer phosphorylated in $\triangle \mathrm{PRP} 4 \mathrm{~K}$ extract, tri-snRNP stability might also be affected, leading to a reduction in $\mathrm{B}$ complex formation. To assay a

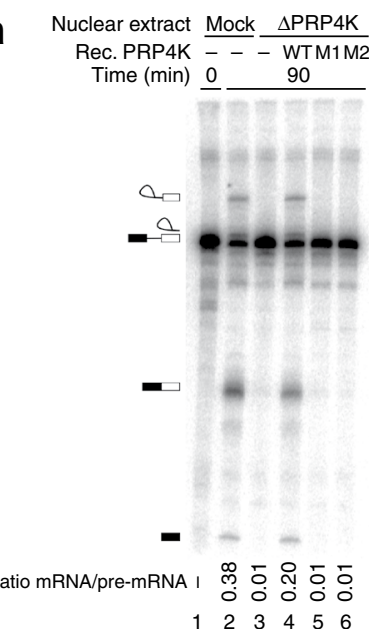

for changes in tri-snRNP stability, we subjected mock-depleted and $\triangle \mathrm{PRP} 4 \mathrm{~K}$ extract to glycerol gradient centrifugation and monitored the distribution of U4/U6-U5 tri-snRNPs by northern blotting. We did not detect a change in the distribution of the U4, U5 or U6 small nuclear RNAs (snRNAs) upon PRP4K depletion (Fig. 5a,b, above). Similarily, the distribution of PRP31 was also not affected (Fig. $\mathbf{5 a}, \mathbf{b}$, below). Thus, the block in $\mathrm{B}$ complex formation is not a consequence of reduced amounts of tri-snRNP in $\triangle \mathrm{PRP} 4 \mathrm{~K}$ extract.

In Figure 4, we analyzed B complex formation on native gels in the presence of heparin, which can 'strip' less stably associated components from the pre-mRNA. Indeed, affinity selection of spliceosomal complexes in the absence of heparin (Fig. 5b) revealed that PRP6 and PRP31 - and thus presumably tri-snRNPscan still associate with the pre-mRNA in $\triangle \mathrm{PRP} 4 \mathrm{~K}$ extract. To test whether tri-snRNPs still dock with A complexes after PRP4K depletion, we performed pull-down experiments with amylose beads after incubating MS2-MBP-bound MINX pre-mRNA under splicing conditions in mock or $\triangle \mathrm{PRP} 4 \mathrm{~K}$ extract for $10 \mathrm{~min}$ to allow $B$ complex formation. We observed nearly identical amounts of co-purified U4, U5 and U6 snRNA in mock-depleted versus $\triangle$ PRP4K extract (Fig. 5c, lanes 3 and 4). This indicates that PRP4K is not required for initial tri-snRNP docking but, rather, for the stable (that is, heparin-resistant) integration of the tri-snRNP during a

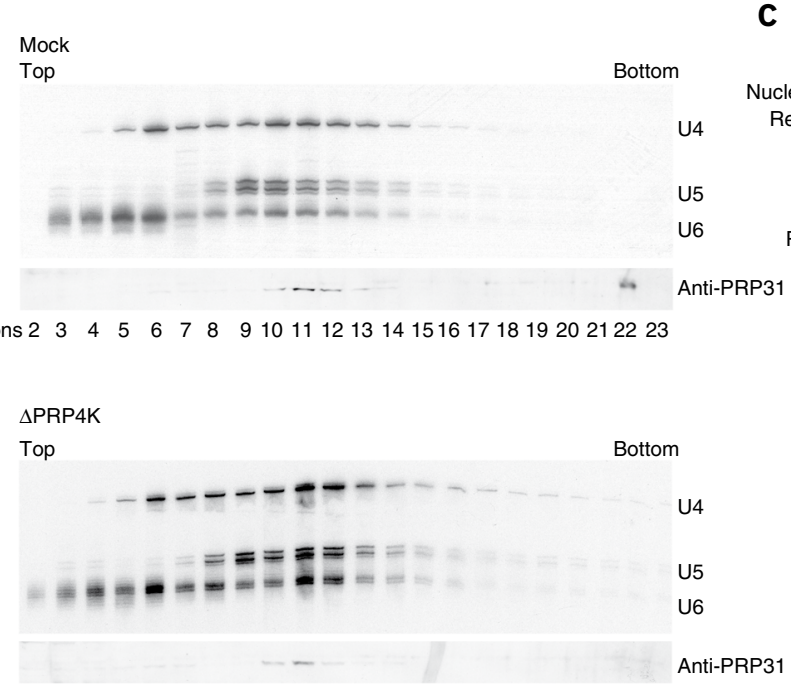

Fractions $2 \quad 3 \quad 4 \quad 5 \quad 6 \quad 7 \quad 8 \quad 9 \quad 1011121314151617181920212223$
Figure 5 Tri-snRNP assembly and stability are not affected by PRP4K depletion. Mock-depleted (a) and PRP4K-depleted ( $\triangle$ PRP4K) (b) nuclear extract were analyzed by glycerol gradient centrifugation. The RNA and protein composition of each gradient fraction were analyzed by northern blotting with probes against U4, U5 and U6 snRNA and by western blotting with antibodies against all forms of human PRP31, respectively. 'Top' and 'bottom' refer to the top or bottom of the centrifuge tube. (c) Spliceosomes were allowed to form on MS2MBP-bound MINX pre-mRNA (lanes 2-5) or MINX pre-mRNA alone (lane 1 ) by incubating under splicing conditions in mock-depleted (lanes 1 and 3) or $\triangle$ PRP4K extract (lanes 4 and 5). In lane 2, ATP was omitted. Complexes were precipitated by affinity selection with amylose beads, and the RNA was recovered, separated by denaturing PAGE and visualized by silver staining. Positions of the MINX pre-mRNA and the U1, U2, U4, U5 and U6 snRNAs are indicated at left. 'Top' and 'bottom' refer to the top or bottom of the centrifuge tube. 
B complex formation. As splicing was inhibited by PRP4K depletion, our results further indicate that although tri-snRNPs can bind to pre-spliceosomes in the absence of PRP4K, the resultant spliceosomal complexes are functionally compromised.

\section{DISCUSSION}

Here we show that the human tri-snRNP proteins PRP6 and PRP31 are phosphorylated after tri-snRNP incorporation into the spliceosomal $\mathrm{B}$ complex, and we provide evidence that both proteins are directly phosphorylated by PRP4 kinase in this complex. Human PRP4K activity is required not only for the phosphorylation of PRP6 and PRP31, but also for the assembly of stable, functional B complexes. This suggests in turn that phosphorylation of these proteins helps to stabilize the interaction of the tri-snRNP with pre-spliceosomes. Taken together, our data provide new insights into the molecular mechanism of action of PRP4K during splicing.

\section{Phosphorylation of PRP6 and PRP31 is dependent on PRP4K}

Our data strongly suggest that human PRP6 and PRP31 are directly phosphorylated by PRP4K during splicing. PRP6 and PRP31 are first phosphorylated after their incorporation into the spliceosomal $\mathrm{B}$ complex, suggesting that their phosphorylation is tightly regulated and probably mediated and/or triggered by a B complex component. Notably, PRP4K is the only kinase that is consistently detected by MS in purified human B complexes. Furthermore, PRP4K phosphorylates human PRP6 (refs. 29,32) and PRP31 (Fig. 3) in vitro, and the phosphorylation of both proteins is greatly reduced in $\triangle \mathrm{PRP} 4 \mathrm{~K}$ extract (Fig. 3). Thus, it is highly likely that both proteins are directly phosphorylated by human PRP4K both in vitro and in vivo. Consistent with the idea that PRP6 and PRP31 are substrates for PRP4K in vivo, the amino acids of human PRP6 and PRP31 that are phosphorylated in extract under splicing conditions (Supplementary Fig. 2) are evolutionarily conserved, except in S. cerevisiae, in which Prp4K is absent.

\section{The role of PRP6 and PRP31 phosphorylation in splicing}

The U5-associated Prp6 protein has a bridging role in both human and yeast U4/U6-U5 tri-snRNPs ${ }^{35,36}$, through its interaction with the U4/U6-associated Prp31 protein ${ }^{36}$. In humans, PRP31 is also required for the formation of tri-snRNPs both in vitro ${ }^{2}$ and in vivo ${ }^{3}$. The identification of an early role for these proteins in higher eukaryotes does not exclude the possibility that they also act at subsequent steps. Indeed, although not required for yeast tri-snRNP formation, S. cerevisiae Prp31 promotes stable association of the tri-snRNP with the pre-mRNA during B complex formation ${ }^{37}$. This is consistent with the idea that human PRP31 also contributes to the stable integration of tri-snRNPs into human B complexes, and our data further suggest that phosphorylation of PRP31 would be required for its putative stabilizing effect. Phosphorylation of PRP31 could stabilize tri-snRNP association with the A complex by enhancing or promoting protein-protein interactions involving this protein. Human PRP31 interacts with the TPR repeat region of PRP6 (ref. 38), and its NOP domain also binds a composite RNP site within the U4/U6 snRNP that consists of the U4-15.5K (also known as NHP2L1) protein and the U4 snRNA ${ }^{39}$. Human PRP31 has not been reported to interact with other spliceosomal proteins. However, it is conceivable that the PRP31-PRP6 interaction, which is crucial for stable tri-snRNP formation, must be enhanced or in some way altered via phosphorylation to ensure tri-snRNP stability within the B complex or to enhance tri-snRNP interactions with A complex components. Indeed, both of these proteins seem to be phosphorylated concomitantly, supporting the idea that a shared function of these proteins is targeted by phosphorylation. Notably, human PRP6 interacts with a number of other spliceosomal proteins, including SNU66 (ref. 38). In budding yeast, Snu66 is required for splicing and seems to interact with one or more components of the $\mathrm{U} 2 \mathrm{snRNP}^{40}$, which is a major subunit of the spliceosomal A complex. Human SNU66 was shown to be essential for $\mathrm{B}$ complex formation in vitro ${ }^{6}$, and it is conceivable that its function is modulated by phosphorylation of its interaction partner, PRP6.

Additionally or alternatively, the phosphorylation of these proteins may be important for more downstream events. For example, phosphorylation of human PRP6 and PRP31 might contribute to the remodeling of tri-snRNP components during spliceosome activation, when the U4/U6 snRNA base pairing interactions are disrupted and U4 snRNA, together with PRP31 and other U4-associated proteins, is destabilized or released from the spliceosome. As decribed above, PRP31 interacts directly with components of the U4/U6 snRNP and also PRP6. PRP6, in turn, contacts several proteins that have key roles during the catalytic activation of the spliceosome, including PRP8 (also known as PRPF8), BRR2 (also known as SNRNP200) and SNU114 (also known as EFTUD2) ${ }^{38}$. Thus, PRP6 and/or PRP31 phosphorylation could potentially modulate the activity of these proteins. Consistent with this idea, S. pombe Prp6 (denoted Prp1) interacts genetically with both Brr2 and Prp8, suggesting a functional relationship between Prp6 and these proteins ${ }^{33}$. PRP31 and PRP6 are destabilized and/or lost from human spliceosomes during catalytic activation, together with other U4/U6 and tri-snRNP proteins ${ }^{41}$, and phosphorylation (or even subsequent dephosphorylation) could potentially be a prerequisite for their release from the spliceosome.

\section{The molecular mechanism of PRP4K action}

Our data reveal that human PRP4K is not required for tri-snRNP stability, nor for its initial docking to the pre-spliceosome (Fig. 5), but instead for stable tri-snRNP integration (Fig. 4). Probable targets of PRP4K that mediate stable tri-snRNP integration are PRP6 and PRP31. However, we cannot exclude the possibility that other targets of this kinase additionally or alternatively have key roles in this process. For example, at least one SR protein (ASF) is phosphorylated in vitro by PRP $4 \mathrm{~K}^{30,31}$, and loss of SR protein phosphorylation upon PRP4K depletion could theoretically contribute to reduced B complex stability. However, other kinases that phosphorylate SR proteins should still be active in $\triangle \mathrm{PRP} 4 \mathrm{~K}$ extract, and individual SR proteins can functionally substitute for each other at this stage ${ }^{9}$. Other proteins involved in tri-snRNP integration during spliceosome assembly-for example, PRP28, SNU66 or SAD1 (refs. 5,6)—could also potentially be substrates of PRP4K. However, at present, the modification status of SNU66 and SAD1 is unclear. Phosphorylation of human PRP28 is required for $\mathrm{B}$ complex formation but is mediated by the kinase SRPK2 (ref. 5).

\section{Multiple phosphorylation events during B complex assembly}

In higher eukaryotes, post-translational modifications act as molecular switches during spliceosome assembly and catalytic activation, and thus have important regulatory roles in the progression of splicing. Our data, together with that from previous studies on PRP28, suggest that tri-snRNP addition during human B complex formation is dependent on multiple phosphorylation events, including phosphorylation of PRP28 (ref. 5) and probably also of PRP6 and PRP31. Thus, this stage of splicing is potentially a 'hot spot' for regulation.

S. cerevisiae lacks Prp4K, and the serine and threonine residues of PRP6 and PRP31 that are phosphorylated in humans are not conserved in S. cerevisiae, suggesting differential requirements for stable tri-snRNP association. In S. cerevisiae, $\operatorname{Prp} 28$ is required for stable tri-snRNP 
addition to the A complex ${ }^{42}$. Phosphorylated PRP28 is required for $\mathrm{B}$ complex formation in humans, but whether it stabilizes tri-snRNP association during this stage is not known ${ }^{5}$. Furthermore, the kinase SRPK2, which is also essential for human B complex formation, is absent in S. cerevisiae. This kinase phosphorylates RS-rich domains that, in higher eukayotes, are typically found in SR proteins and other spliceosomal proteins (including PRP28) but are nearly absent in S. cerevisiae. Indeed, there seems to be generally fewer phosphorylation events during splicing in S. cervisiae, and thus fewer regulatory targets or switches. This is consistent with the lack of alternative splicing events in yeast, and also with the more flexible nature of interactions among spliceosomal components in higher eukaryotes, which may therefore be more susceptible to fine-tuning by post-translational modifications.

\section{METHODS}

Methods and any associated references are available in the online version of the paper at http://www.nature.com/nsmb/.

Note: Supplementary information is available on the Nature Structural \& Molecular Biology website.

\section{ACKNOWLEDGMENTS}

We are grateful to T. Conrad and H. Kohansal for help in preparing HeLa cell nuclear extract. We would also like to thank C. Girard for critical discussions, K. Hartmuth (Max Planck Insitute (MPI) for Biophysical Chemistry) for providing phosphospecific SF3b155 antibody and S. Trowitzsch and G. Weber (MPI for Biophysical Chemistry) for providing purified human U4/U6-U5 tri-snRNPs. This work was supported by grants from the Deutschen Forschungsgemeinschaft, the European Commission (EURASNET-518238), Fonds der Chemischen Industrie and the Ernst Jung Stiftung to R.L. and a Young Investigator Programme grant from EURASNET to H.U.

\section{AUTHOR CONTRIBUTIONS}

M.S., H.-H.H., H.U. and R.L. designed the research; M.S. and H.-H.H. performed the research; R.G. provided anti-PRP4K antibodies; M.S., H.-H.H., C.L.W., H.U. and R.L. analyzed the data; M.S., C.L.W. and R.L. wrote the paper.

\section{COMPETING INTERESTS STATEMENT}

The authors declare no competing financial interests.

Published online at http://www.nature.com/nsmb/.

Reprints and permissions information is available online at http://npg.nature.com/ reprintsandpermissions/.

1. Will, C.L. \& Lührmann, R. Spliceosome structure and function. in The RNA World 3rd edn (eds. Gesteland, R.F., Cech, T.R. \& Atkins, J.F.) 369-400 (Cold Spring Harbor Laboratory Press, Cold Spring Harbor, 2006).

2. Makarova, O.V., Makarov, E.M., Liu, S., Vornlocher, H.P. \& Lührmann, R. Protein $61 \mathrm{~K}$, encoded by a gene (PRPF31) linked to autosomal dominant retinitis pigmentosa, is required for U4/U6*U5 tri-snRNP formation and pre-mRNA splicing. EMBO J. 21, 1148-1157 (2002).

3. Schaffert, N., Hossbach, M., Heintzmann, R., Achsel, T. \& Lührmann, R. RNAi knockdown of hPRP31 leads to an accumulation of U4/U6 di-snRNPs in Cajal bodies. EMBO J. 23, 3000-3009 (2004).

4. Vithana, E.N. et al. A human homolog of yeast pre-mRNA splicing gene, PRP31, underlies autosomal dominant retinitis pigmentosa on chromosome 19q13.4 (RP11). Mol. Cell 8, 375-381 (2001).

5. Mathew, R. et al. Phosphorylation of human PRP28 by SRPK2 is required for integration of the U4/U6-U5 tri-snRNP into the spliceosome. Nat. Struct. Mol. Biol. 15, 435-443 (2008).

6. Makarova, O.V., Makarov, E.M. \& Lührmann, R. The 65 and 110 kDa SR-related proteins of the U4/U6.U5 tri-snRNP are essential for the assembly of mature spliceosomes. EMBO J. 20, 2553-2563 (2001).

7. Meister, G. et al. SMNrp is an essential pre-mRNA splicing factor required for the formation of the mature spliceosome. EMBO J. 20, 2304-2314 (2001).

8. Rappsilber, J., Ajuh, P., Lamond, A.I. \& Mann, M. SPF30 is an essential human splicing factor required for assembly of the U4/U5/U6 tri-small nuclear ribonucleoprotein into the spliceosome. J. Biol. Chem. 276, 31142-31150 (2001).

9. Roscigno, R.F. \& Garcia-Blanco, M.A. SR proteins escort the U4/U6.U5 tri-snRNP to the spliceosome. RNA 1, 692-706 (1995).

10. Mermoud, J.E., Cohen, P. \& Lamond, A.I. Ser/Thr-specific protein phosphatases are required for both catalytic steps of pre-mRNA splicing. Nucleic Acids Res. 20, 5263-5269 (1992).

11. Tazi, J. et al. Thiophosphorylation of U1-70K protein inhibits pre-mRNA splicing. Nature 363, 283-286 (1993).
12. Cao, W., Jamison, S.F. \& Garcia-Blanco, M.A. Both phosphorylation and dephosphorylation of ASF/SF2 are required for pre-mRNA splicing in vitro. RNA 3 1456-1467 (1997)

13. Xiao, S.H. \& Manley, J.L. Phosphorylation of the ASF/SF2 RS domain affects both protein-protein and protein-RNA interactions and is necessary for splicing. Genes Dev. 11, 334-344 (1997).

14. Murray, H.L. \& Jarrell, K.A. Flipping the switch to an active spliceosome. Cell 96, 599-602 (1999).

15. Shi, Y., Reddy, B. \& Manley, J.L. PP1/PP2A phosphatases are required for the second step of Pre-mRNA splicing and target specific snRNP proteins. Mol. Cell 23, 819-829 (2006).

16. Manley, J.L. \& Tacke, R. SR proteins and splicing control. Genes Dev. 10 1569-1579 (1996).

17. Graveley, B.R. Sorting out the complexity of SR protein functions. RNA 6 1197-1211 (2000).

18. Woppmann, A., Patschinsky, T., Bringmann, P., Godt, F. \& Lührmann, R. Characterisation of human and murine snRNP proteins by two-dimensional gel electrophoresis and phosphopeptide analysis of U1-specific $70 \mathrm{~K}$ protein variants. Nucleic Acids Res. 18, 4427-4438 (1990).

19. Wang, C. et al. Phosphorylation of spliceosomal protein SAP 155 coupled with splicing catalysis. Genes Dev. 12, 1409-1414 (1998).

20. Fetzer, S., Lauber, J., Will, C.L. \& Lührmann, R. The [U4/U6.U5] tri-snRNP-specific $27 \mathrm{~K}$ protein is a novel SR protein that can be phosphorylated by the snRNPassociated protein kinase. RNA 3, 344-355 (1997).

21. Sanford, J.R., Longman, D. \& Caceres, J.F. Multiple roles of the SR protein family in splicing regulation. Prog. Mol. Subcell. Biol. 31, 33-58 (2003).

22. Colwill, K. et al. The Clk/Sty protein kinase phosphorylates SR splicing factors and regulates their intranuclear distribution. EMBO J. 15, 265-275 (1996).

23. Rossi, F. et al. Specific phosphorylation of SR proteins by mammalian DNA topoisomerase I. Nature 381, 80-82 (1996).

24. Gui, J.F., Tronchere, H., Chandler, S.D. \& Fu, X.D. Purification and characterization of a kinase specific for the serine- and arginine-rich pre-mRNA splicing factors. Proc. Natl. Acad. Sci. USA 91, 10824-10828 (1994).

25. Kuroyanagi, N., Onogi, H., Wakabayashi, T. \& Hagiwara, M. Novel SR-proteinspecific kinase, SRPK2, disassembles nuclear speckles. Biochem. Biophys. Res. Commun. 242, 357-364 (1998).

26. Wang, J., Xiao, S.H. \& Manley, J.L. Genetic analysis of the SR protein ASF/SF2: interchangeability of RS domains and negative control of splicing. Genes Dev. 12, 2222-2233 (1998).

27. Rosenberg, G.H., Alahari, S.K. \& Kaufer, N.F. prp4 from Schizosaccharomyces pombe, a mutant deficient in pre-mRNA splicing isolated using genes containing artificial introns. Mol. Gen. Genet. 226, 305-309 (1991).

28. Hanks, S.K. \& Hunter, T. Protein kinases 6. The eukaryotic protein kinase superfamily: kinase (catalytic) domain structure and classification. FASEB J. 9 576-596 (1995).

29. Dellaire, G. et al. Mammalian PRP4 kinase copurifies and interacts with components of both the U5 snRNP and the N-CoR deacetylase complexes. Mol. Cell. Biol. 22 5141-5156 (2002).

30. Gross, T. et al. Functional analysis of the fission yeast Prp4 protein kinase involved in pre-mRNA splicing and isolation of a putative mammalian homologue. Nucleic Acids Res. 25, 1028-1035 (1997).

31. Kojima, T., Zama, T., Wada, K., Onogi, H. \& Hagiwara, M. Cloning of human PRP4 reveals interaction with Clk1. J. Biol. Chem. 276, 32247-32256 (2001).

32. Schwelnus, W. et al. Fission yeast Prp4p kinase regulates pre-mRNA splicing by phosphorylating a non-SR-splicing factor. EMBO Rep. 2, 35-41 (2001).

33. Bottner, C.A., Schmidt, H., Vogel, S., Michele, M. \& Kaufer, N.F. Multiple genetic and biochemical interactions of Brr2, Prp8, Prp31, Prp1 and Prp4 kinase suggest a function in the control of the activation of spliceosomes in Schizosaccharomyces pombe. Curr. Genet. 48, 151-161 (2005).

34. Montembault, E., Dutertre, S., Prigent, C. \& Giet, R. PRP4 is a spindle assembly checkpoint protein required for MPS1, MAD1, and MAD2 localization to the kinetochores. J. Cell Biol. 179, 601-609 (2007)

35. Galisson, F. \& Legrain, P. The biochemical defects of prp4-1 and prp6-1 yeast splicing mutants reveal that the PRP6 protein is required for the accumulation of the [U4/U6.U5] tri-snRNP. Nucleic Acids Res. 21, 1555-1562 (1993).

36. Makarov, E.M., Makarova, O.V., Achsel, T. \& Lührmann, R. The human homologue of the yeast splicing factor prp6p contains multiple TPR elements and is stably associated with the U5 snRNP via protein-protein interactions. J. Mol. Biol. 298, 567-575 (2000).

37. Weidenhammer, E.M., Ruiz-Noriega, M. \& Woolford, J.L. Jr. Prp31p promotes the association of the U4/U6 6 U5 tri-snRNP with prespliceosomes to form spliceosomes in Saccharomyces cerevisiae. Mol. Cell. Biol. 17, 3580-3588 (1997).

38. Liu, S., Rauhut, R., Vornlocher, H.P. \& Lührmann, R. The network of protein-protein interactions within the human U4/U6.U5 tri-snRNP. RNA 12, 1418-1430 (2006).

39. Liu, S. et al. Binding of the human Prp31 Nop domain to a composite RNA-protein platform in U4 snRNP. Science 316, 115-120 (2007).

40. Gottschalk, A. et al. Identification by mass spectrometry and functional analysis of novel proteins of the yeast [U4/U6.U5] tri-snRNP. EMBO J. 18, 4535-4548 (1999).

41. Bessonov, S., Anokhina, M., Will, C.L., Urlaub, H. \& Lührmann, R. Isolation of an active step I spliceosome and composition of its RNP core. Nature 452, 846-850 (2008).

42. Staley, J.P. \& Guthrie, C. An RNA switch at the $5^{\prime}$ splice site requires ATP and the DEAD box protein Prp28p. Mol. Cell 3, 55-64 (1999). 


\section{ONLINE METHODS}

Immunizations and western blot analyses. To generate phosphospecific human PRP6 or PRP31 antibodies, we immunized rabbits with the following phosphopeptides (phosphorylated $\left({ }^{\mathrm{P}}\right)$ threonines and serines are indicated): KGYLT $^{\mathrm{P}}$ DLNS $^{\mathrm{P}}$ (PRP6 residues 271-279) or VYGGKS ${ }^{\mathrm{P}} \mathrm{T}^{\mathrm{P}}$ IRDRSSGT $^{\mathrm{P}}$ ASSVA (PRP31 residues 434-453). To obtain phosphospecific SF3B155 antibodies, we immunized rabbits with the phosphopeptide HGSGWAET ${ }^{\mathrm{P}}$ RRTDRGG (SF3B155 residues 306-320). Before use, we passed the phosphospecific antisera over a column containing nonphosphorylated cognate peptide, to remove nonphosphospecific antibodies. For western blotting, we separated proteins on a $10 \%$ SDSpolyacrylamide gel, transferred them to nitrocellulose and analyzed them using an enhanced chemoluminescence (ECL) detection kit (Pierce) with the following antibodies: Prp4K ${ }^{34}$, PRP6 (ref. 36), PRP31(ref. 2), SNU66 (ref. 6), phosphospecific SF3B155, phosphospecific PRP6 or phosphospecific PRP31.

In vitro splicing. We prepared HeLa nuclear extract as described ${ }^{43}$. Splicing reactions contained $2-10 \mathrm{nM}^{32} \mathrm{P}$-labeled MINX pre-mRNA ${ }^{44}$ or $\beta$-globin premRNA, $65 \mathrm{mM} \mathrm{KCl}, 3 \mathrm{mM} \mathrm{MgCl} 2,2 \mathrm{mM}$ ATP, $20 \mathrm{mM}$ creatine phosphate and $40 \%(\mathrm{v} / \mathrm{v}) \mathrm{HeLa}$ nuclear extract. We incubated the reactions at $30^{\circ} \mathrm{C}$ for the indicated time periods. We analyzed the pre-mRNA and splicing intermediates and products by denaturing PAGE and the spliceosomal complexes by native PAGE, as described ${ }^{2}$.

Purification of tri-snRNPs and spliceosomal B complexes. We obtained human U4/U6-U5 tri-snRNPs by anti- ${ }_{3} \mathrm{G}$ immunoaffinity chromatography and subsequent glycerol gradient centrifugation ${ }^{45}$. We purified the human spliceosomal $\mathrm{B}$ complexes from in vitro splicing reactions by the MS2 affinity-selection method ${ }^{46}$. Briefly, we incubated the MINX pre-mRNA substrate (containing MS2 aptamers bound by MBP-MS2 protein) for 5-10 min under splicing conditions to allow $\mathrm{B}$ complex formation. We isolated the B complexes using glycerol gradient centrifugation followed by amylose affinity selection of gradient fractions containing the B complex. In some cases (as indicated), spliceosomal complexes were affinity selected directly from the splicing reaction.

Immunodepletion of PRP4 kinase. To immunodeplete human PRP4K, we first adjusted the $\mathrm{NaCl}$ concentration of HeLa nuclear extract to $1 \mathrm{M}$. We then added the extract to Protein A-Sepharose beads bound by anti-PRP4K antibodies ${ }^{34}$ and incubated the mixture with end-over-end rotation for $1 \mathrm{~h}$ at $4{ }^{\circ} \mathrm{C}$. We then repeated the procedure with a second aliquot of antibody-bound protein ASepharose beads. We recovered the nuclear extract and dialyzed it twice for $1.5 \mathrm{~h}$ at $4{ }^{\circ} \mathrm{C}$ against Roeder D buffer (20 mM HEPES-KOH (pH 7.9), $100 \mathrm{mM} \mathrm{KCl}$, 1.5. $\mathrm{mM} \mathrm{MgCl} 2,0.2 \mathrm{mM}$ EDTA ( $\mathrm{pH} 8.0$ ), $1 \mathrm{mM}$ dithioerythritol, $0.5 \mathrm{mM}$ PMSF, 20\% (v/v) glycerol). Mock-depleted extract was prepared in an identical manner except that the antibody was omitted. For splicing-complementation studies, we added $450 \mathrm{ng}$ of recombinant wild-type or mutant human PRP4K to splicing reactions containing $\triangle \mathrm{PRP} 4 \mathrm{~K}$ nuclear extract. To analyze the integrity of U4/U6-U5 tri-snRNPs after PRP4K immunodepletion, mock-depleted or $\triangle \mathrm{PRP} 4 \mathrm{~K}$ extract was fractionated on a $10-30 \%(\mathrm{v} / \mathrm{v})$ glycerol gradient by centrifugation for $2 \mathrm{~h}$ at $488,576 \mathrm{~g}\left(r_{\max }\right)$. We determined the distribution of $\mathrm{U} 4, \mathrm{U} 5$ and U6 snRNAs across the gradient by northern analysis, and that of tri-snRNP-associated proteins by western analysis.

Expression of recombinant PRP4 kinase. His-tagged, human PRP4K was expressed in the baculovirus system. We obtained the human PRP4 kinase cDNA sequence from Invitrogen (CloneID: IOH45196) in a Gateway entry vector and transformed it into the pDEST 10 vector with an N-terminal His-tag, according to the manufacturer's protocol. We produced the PRP4K mutants M1 (K717I) and M2 (D815A) by site-directed mutagenesis of the latter vector and verified them via sequencing. Recombination was performed in Escherichia coli strain $\mathrm{DH} 10 \mathrm{Ba}$, and the resulting PRP4K-containing bacmid was purified according to the manufacturer's protocol. We produced viral stocks in SF9 insect cells. For purification of recombinant PRP4K, we harvested High Five insect cells $48 \mathrm{~h}$ after transfection with baculovirus. We lysed the cells in suspension buffer $(50 \mathrm{mM}$ Tris- $\mathrm{HCl}$ ( $\mathrm{pH}$ 8.0), $1 \mathrm{M} \mathrm{NaCl}, 10 \mathrm{mM}$ imidazole) by repeated freezing and thawing. The cleared lysate was applied to a Ni-agarose column, washed with buffer $\mathrm{W}$ (50 mM Tris- $\mathrm{HCl}$ (pH 8.0), $1 \mathrm{M} \mathrm{NaCl}, 20 \mathrm{mM}$ imidazole), and purified PRP4K was eluted with elution buffer (50 mM Tris- $\mathrm{HCl}(\mathrm{pH} 8.0), 150 \mathrm{mM} \mathrm{NaCl}$, $400 \mathrm{mM}$ imidazole). Purified PRP4K was dialyzed against Roeder D buffer.

In vitro kinase assays. We assessed the activity of recombinant wild-type or mutant human PRP4K (as indicated) by incubating $450 \mathrm{ng}$ of purified kinase with either $100 \mu \mathrm{g}$ of phosphorylated or nonphosphorylated PRP31 peptide (residues 434-453; see above) or $7 \mu$ g of recombinant MBP-PRP31 (ref. 47) or MS2-MBP in the presence of $\left(\gamma^{32} \mathrm{P}\right)$ ATP for $10 \mathrm{~min}$ at $30^{\circ} \mathrm{C}$. We analyzed the peptides by thin-layer chromatography and the proteins by $10 \%$ SDS-PAGE, followed by autoradiography. We performed immunoprecipitation of phosphorylated proteins essentially as described 5 .

Identification of phosphorylated amino acids by mass spectrometry. Proteins recovered from purified B complexes were separated by SDS-PAGE and stained with Coomassie Brilliant Blue. We cut entire lanes of the gel into multiple slices, and the proteins were digested in-gel with trypsin, extracted as described ${ }^{48}$ and dried under vacuum. Phosphopeptides were enriched by titanium dioxide $\left(\mathrm{TiO}_{2}\right.$; GL Sciences) as described ${ }^{49}$. For ESI MS/MS analysis, we first loaded the enriched peptides onto an in-house-packed C18 trap column $(15 \times 0.15 \mathrm{~mm}$, Nucleosil 100-5 C18; Macherey-Nagel). The retained peptides were eluted and separated on an analytical C18 capillary column $(300 \times 0.075 \mathrm{~mm}$, Nucleosil 100-5 C18) at a flow rate of $300 \mathrm{nl} \mathrm{min}{ }^{-1}$, with a gradient from $7.5 \%$ to $37.5 \%(\mathrm{v} / \mathrm{v}$ ) acetonitrile in $0.1 \%(\mathrm{v} / \mathrm{v})$ formic acid for $60 \mathrm{~min}, 120 \mathrm{~min}$ or $240 \mathrm{~min}$, using an Agilent Technologies 1100 nano-flow LC system coupled to a Q-TOF mass spectrometer (Waters Q-Tof Ultim). We recorded MS and MS/MS spectra under standard conditions and searched used MASCOT v2.2 against the NCBI nr database limited to human taxonomy with the following criteria: peptide mass tolerance, $50 \mathrm{ppm}$; MS/MS ion mass tolerance, $0.25 \mathrm{Da}$; allow up to two missed cleavages; variable modifications were phosphorylation of serine, threonine and tyrosine, methionine oxidation and cysteine carboxyamidomethylation. All fragment spectra of phosphorylated peptides were inspected manually for the presence of $69-\mathrm{Da}$ dihydroalanine (phosphorylated serine after loss of $\mathrm{H}_{3} \mathrm{PO}_{4}$ ) and 83-Da dihydroaminobutyric acid (phosphorylated threonine after loss of $\mathrm{H}_{3} \mathrm{PO}_{4}$ ).

43. Dignam, J.D., Lebovitz, R.M. \& Roeder, R.G. Accurate transcription initiation by RNA polymerase II in a soluble extract from isolated mammalian nuclei. Nucleic Acids Res. 11, 1475-1489 (1983).

44. Zillmann, M., Zapp, M.L. \& Berget, S.M. Gel electrophoretic isolation of splicing complexes containing U1 small nuclear ribonucleoprotein particles. Mol. Cell. Biol. 8, 814-821 (1988).

45. Sander, B. et al. Organization of core spliceosomal components U5 snRNA loop and U4/U6 Di-snRNP within U4/U6.U5 Tri-snRNP as revealed by electron cryomicroscopy. Mol. Cell 24, 267-278 (2006).

46. Deckert, J. et al. Protein composition and electron microscopy structure of affinitypurified human spliceosomal B complexes isolated under physiological conditions. Mol. Cell. Biol. 26, 5528-5543 (2006).

47. Schultz, A., Nottrott, S., Hartmuth, K. \& Lührmann, R. RNA structural requirements for the association of the spliceosomal hPRP31 protein with the U4 and U4atac small nuclear ribonucleoproteins. J. Biol. Chem. 281, 28278-28286 (2006).

48. Shevchenko, A., Wilm, M., Vorm, O. \& Mann, M. Mass spectrometric sequencing of proteins silver-stained polyacrylamide gels. Anal. Chem. 68, 850-858 (1996).

49. Larsen, M.R., Thingholm, T.E., Jensen, O.N., Roepstorff, P. \& Jørgensen, T.J. Highly selective enrichment of phosphorylated peptides from peptide mixtures using titanium dioxide microcolumns. Mol. Cell. Proteomics 4, 873-886 (2005). 